\title{
Angelica Polysaccharide Ameliorates Sepsis-Induced Acute Lung Injury through Inhibiting NLRP3 and NF- $\kappa$ B Signaling Pathways in Mice
}

\author{
Yan Zhu $\mathbb{D}^{1},{ }^{1}$ Taocheng Meng, ${ }^{1}$ Aichen Sun $\mathbb{D}^{2},{ }^{2}$ Jintao Li $\mathbb{D}^{1},{ }^{1}$ and Jinlai Li ${ }^{1}$ \\ ${ }^{1}$ Department of ICU, Zaozhuang Municipal Hospital (Jining Medical College Affiliated Hospital), Zaozhuang, \\ Shandong 277102, China \\ ${ }^{2}$ Department of Orthopedic, Zaozhuang Municipal Hospital (Jining Medical College Affiliated Hospital), Zaozhuang, \\ Shandong 277102, China \\ Correspondence should be addressed to Aichen Sun; sunaichen112@sohu.com and Jintao Li; budcome@126.com
}

Received 13 August 2020; Revised 21 December 2020; Accepted 24 December 2020; Published 19 February 2021

Academic Editor: Tânia Silvia Fröde

Copyright ( 2021 Yan Zhu et al. This is an open access article distributed under the Creative Commons Attribution License, which permits unrestricted use, distribution, and reproduction in any medium, provided the original work is properly cited.

Objective. This study aimed to explore the role of angelica polysaccharide (AP) in sepsis-induced acute lung injury (ALI) and its underlying molecular mechanism. Methods. A sepsis model of cecal ligation and puncture (CLP) in male BALB/C mice was used. Then, $24 \mathrm{~h}$ after CLP, histopathological changes in lung tissue, lung wet/dry weight ratio, and inflammatory cell infiltration were analyzed. Next, levels of inflammatory cytokines (tumor necrosis factor- $\alpha$ (TNF- $\alpha$ ), interleukin (IL)-1 $\beta$, IL-6, and IL-18), as well as the activity of myeloperoxidase (MPO), malondialdehyde (MDA), superoxide dismutase (SOD), and glutathione (GSH), were measured to assess the role of AP. The protein expression of NF- $\kappa \mathrm{B}$ p65, p-NF- $\kappa \mathrm{B}$ p 65 , I $\kappa \mathrm{B} \alpha, \mathrm{p}$-I $\kappa \mathrm{B} \alpha$, nucleotidebinding domain- (NOD-) like receptor protein 3 (NLRP3), ASC, and caspase-1 was detected by western blot. In addition, the expression of p-NF- $\kappa \mathrm{B}$ p65 and NLRP3 was detected by immunohistochemistry. Results. AP treatment ameliorated CLPinduced lung injury and lung edema, as well as decreased the number of total cells, neutrophils, and macrophages in bronchoalveolar lavage fluid (BALF). AP reduced the levels of TNF- $\alpha$, IL-1 $\beta$, IL-6, and IL-18 in BALF, as well as in serum. Moreover, AP decreased MPO activity and MDA content, whereas increased SOD and GSH levels. AP inhibited the expression of p-NF- $\kappa \mathrm{B}$ p65, p-I $\kappa \mathrm{B} \alpha$, NLRP3, ASC, and caspase-1, while promoted $\mathrm{I} \kappa \mathrm{B} \alpha$ expression. Conclusion. This study demonstrated that AP exhibits protective effects against sepsis-induced ALI by inhibiting NLRP3 and NF- $\kappa$ B signaling pathways in mice.

\section{Introduction}

Sepsis, a serious systemic inflammatory response syndrome that causes a harmful systemic inflammatory response to an infection, is related to fatal organ dysfunction and has a high mortality rate [1]. It can lead to multiple tissue and organ injuries, and even death. Patients with sepsis are at great risk for acute lung injury (ALI) due to pulmonary susceptibility [2]. Despite advances being made in management strategies, the mortality rate of patients with ALI induced by sepsis remains high. Therefore, it is important to search for novel therapeutics for the treatment of sepsis-induced ALI.
Angelica sinensis is a perennial Umbelliferae herb and is broadly used as a traditional Chinese herbal medicine or food in Asian countries, such as China, Japan, and Korea [3]. Angelica polysaccharide (AP), one of the active components of Angelica sinensis, plays an important role in enhancing immunity and has antivirus, antitumor, anti-inflammatory, antioxidant, and antiapoptosis activities [4-7]. Previous studies have indicated that AP may alleviate LPS-induced inflammation and apoptosis in PC12 cells by inhibiting COX-1 expression [5]. However, whether AP has therapeutic effects against sepsis-induced ALI is currently uncertain. 
The nuclear factor kappa B $(\mathrm{NF}-\kappa \mathrm{B})$ pathway and nucleotide-binding domain- (NOD-) like receptor protein 3 (NLRP3) inflammasome, as important inflammatory pathways, are reported to play important roles in various diseases $[8,9]$. NF- $\kappa \mathrm{B}$ is an important cell transcription regulator, which can regulate various cytokines participating in the inflammatory response [10]. The NLRP3 inflammasome is an important part of inherent immunity, consisting of NLRP3, the adaptor protein ASC, and caspase-1 [11]. NLRP3 inflammasome activation leads to caspase- 1 activation, which cleaves the inflammatory factor precursors of interleukin- (IL-) $1 \beta$ and IL-18 to accelerate their maturation and release [12]. IL-1 $\beta$ and IL-18 then induce the release of other inflammatory factors, which further amplify the inflammatory response [13]. An increasing number of studies have confirmed that the NF- $\kappa$ B pathway and NLRP3 inflammasome play vital roles in the therapy of ALI $[14,15]$.

In the present study, we investigated the effect of AP on sepsis-induced ALI and its related molecular mechanisms. Our data confirmed that AP could ameliorate sepsisinduced ALI in mice by inhibiting NLRP3 and NF- $\kappa \mathrm{B}$ signaling pathways. Our findings are beneficial for further understanding the protective effect of AP against sepsisinduced ALI.

\section{Materials and Methods}

2.1. Animals. Male BALB/C mice (20-24 g, six-to-eight weeks old) were purchased from Beijing Vital River Bioscience Co., Ltd (Beijing, China), and the mice were placed in a temperature- and light-controlled room $\left(22 \pm 2^{\circ} \mathrm{C}, 12 \mathrm{~h}\right.$ cycle $)$ with a humidity of $55 \pm 5 \%$. The mice were fed food and water ad libitum. All studies involving animals were approved by the ethics committee of our hospital and conducted in accordance with the National Institutes of Health Guide for the Care and Use of Laboratory Animals.

2.2. Animal Model of Sepsis. The sepsis-induced ALI model was established by cecal ligation and puncture (CLP) surgery, as previously described with minor modifications [16]. Simply, mice were fasted for $12 \mathrm{~h}$ before surgery and anesthetized using pentobarbital sodium (intraperitoneally, $50 \mathrm{mg} / \mathrm{kg}$ ). A $2-\mathrm{cm}$ abdominal midline incision was made, and the cecum was exposed. Subsequently, the cecum was isolated and ligated below the ileocecal valve. Afterward, the cecum between the ligation site and the end of the cecum was punctured using a 22-gauge needle. A small amount of faeces was squeezed through the puncture wound. The cecum was then repositioned into the abdominal cavity, and the abdominal incision was sutured with surgical sutures. Sham-operated mice underwent the same procedure without CLP.

2.3. Experimental Protocols. The mice were randomly divided into five groups $(n=10)$ : Sham group, CLP group, CLP+AP $(25 \mathrm{mg} / \mathrm{kg})$ group, CLP+AP $(50 \mathrm{mg} / \mathrm{kg})$ group, and CLP $+\mathrm{AP}(100 \mathrm{mg} / \mathrm{kg})$ group. Mice in the CLP+AP group were given AP by intraperitoneally $12 \mathrm{~h}$ before CLP, and those in Sham group were given an equal volume of normal saline (NS). Mice were sacrificed $24 \mathrm{~h}$ after CLP. AP was supplied by Ci Yuan Biotechnology Co., Ltd. Shanxi (Xian, China). Lung histological changes and lung wet/dry weight ratios were determined to assess the AP effective concentration for further experiments.

2.4. Lung Wet/Dry Ratio. The lung wet/dry weight ratio was used to assess lung edema. Briefly, the lung tissue was excised and rinsed with saline. After that, the tissues were weighed, and wet weight was recorded. Then, the fresh lung tissues were dried in an $80^{\circ} \mathrm{C}$ oven for at least $48 \mathrm{~h}$ to obtain the dry weight as follows: lung wet/dry ratio = wet weight/dry weight.

\subsection{Bronchoalveolar Lavage Fluid (BALF) Collection and Cell} Count. The trachea was exposed and opened. The BALF was collected via injection and retraction of $1.5 \mathrm{~mL}$ PBS three times. Subsequently, the collected BALF was centrifuged for $10 \mathrm{~min}$ at $3000 \mathrm{rpm}$ at $4^{\circ} \mathrm{C}$. The supernatants were stored at $-80^{\circ} \mathrm{C}$ for cytokine detection using an enzyme-linked immunosorbent assay (ELISA) kit. The cell precipitate was resuspended in $100 \mu \mathrm{L}$ of PBS, and the total cell numbers in BALF were measured using a hemacytometer. Moreover, neutrophil and macrophage cell numbers were evaluated using the Wright-Giemsa staining method.

2.6. ELISA. The retrobulbar venous plexus blood was collected and centrifuged at $3000 \mathrm{rpm}$ for $10 \mathrm{~min}$. The levels of tumor necrosis factor- (TNF-) $\alpha$, interleukin (IL)- $1 \beta$, and IL-6 in BALF and serum were determined using the corresponding ELISA kit (R\&D systems, Minneapolis, MN, USA) in accordance with the manufacturer's instructions.

2.7. Oxidative Stress Assay. According to the instructions of the experimental manual, the lung tissues were homogenized. The levels of myeloperoxidase (MPO), malondialdehyde (MDA), superoxide dismutase (SOD), and glutathione (GSH) were detected using the corresponding test kit supplied by Jiancheng Bioengineering Institute (Nanjing, China).

2.8. Hematoxylin-Eosin (HE) Staining. To evaluate histopathological changes, the lung tissue was removed and fixed in $4 \%$ formaldehyde for $24 \mathrm{~h}$. Thereafter, the lung tissue was routinely dehydrated in a concentration series of alcohol, embedded in paraffin, and cut into $5 \mu \mathrm{m}$ thick sections. The sections were deparaffinized with xylene, dehydrated with ethanol of different gradient concentrations, and then stained with $\mathrm{HE}$ staining for $10 \mathrm{~min}$ at room temperature. Finally, pathological changes in the lung tissues were observed under an optical microscope. Histopathological changes in lung tissue were evaluated using a semiquantitative scoring method. The histological score of the lung tissue was based on alveolar congestion, hemorrhage, inflammatory cell infiltration, and alveolar wall thickness, and graded from 0 (normal) to 4 (severe).

2.9. Immunohistochemistry Analysis. Lung tissue sections $(5 \mu \mathrm{m})$ were incubated with primary rabbit monoclonal antibodies against p-NF- $\kappa \mathrm{B}$ p65 (1:100, ab86299, Abcam, UK) and NLRP3 (1:200, ab214185, Abcam, UK) overnight at 


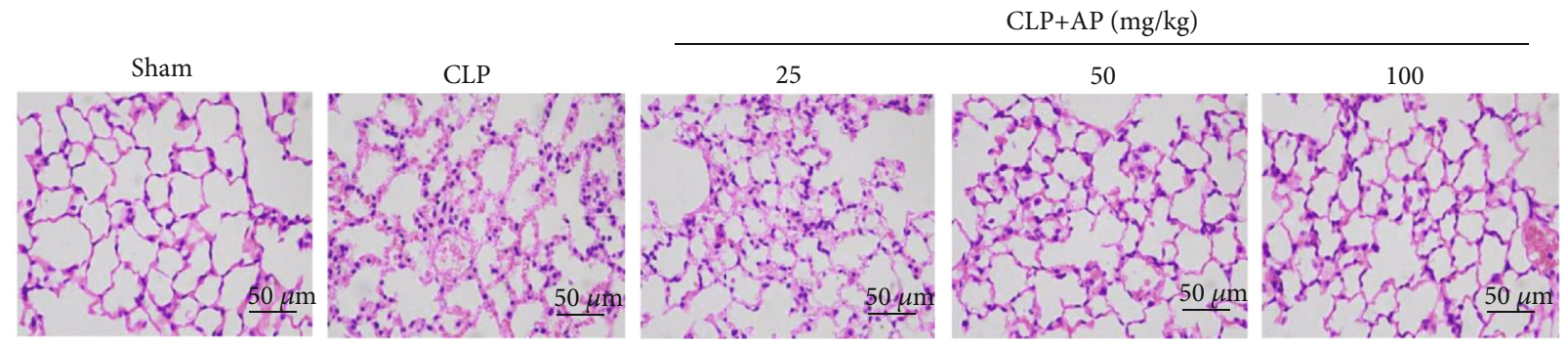

(a)

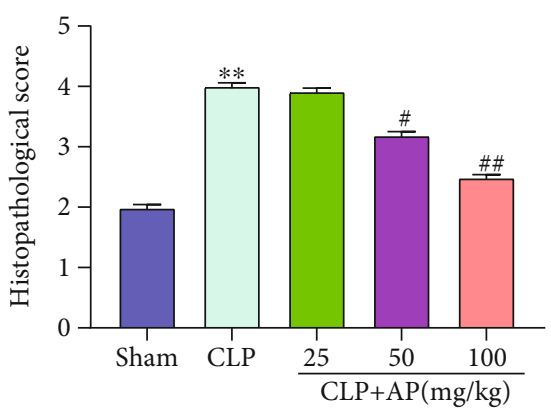

(b)

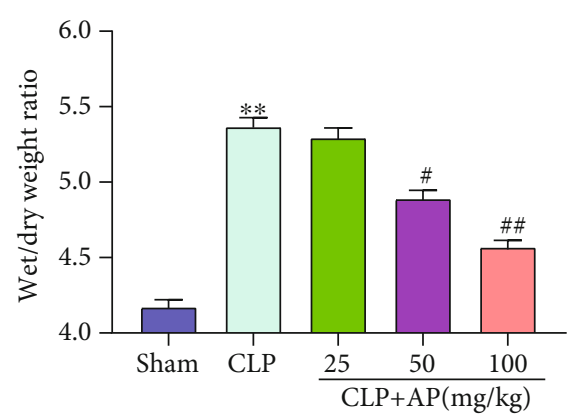

(c)

FIGURE 1: AP protected mice against sepsis-induced ALI and lung edema. (a) Pathological changes in the lung tissues observed by HE staining (100x). (b) Histological score of lung tissue. (c) The lung wet weight/dry weight ratio was assessed among experimental groups. ${ }^{* *} P<0.01$, vs. Sham group; ${ }^{\#} P<0.05,{ }^{\# \#} P<0.01$, vs. CLP group.

$4^{\circ} \mathrm{C}$ and then incubated with alkaline phosphataseconjugated secondary antibody at room temperature for $2 \mathrm{~h}$. After staining with 3, 3' -diaminobenzidine (DAB, KeyGen, Nanjing, China) and counterstaining with hematoxylin, the mean integrated optical density of p-NF- $\kappa \mathrm{B}$ p 65 and NLRP3 was visualized under an optical microscope.

2.10. Western Blot Analysis. The total proteins of lung tissues were extracted using protein extraction kits following the manufacturer's instructions. First, $50 \mu \mathrm{g}$ of denatured protein was loaded onto $10 \%$ sodium dodecyl sulfate-polyacrylamide gel electrophoresis and then transferred onto polyvinylidene fluoride membranes (Millipore, Bedford, MA, USA). The membranes were blocked with $5 \%$ skimmed milk in TBST, followed by overnight incubation at $4^{\circ} \mathrm{C}$ with the corresponding primary antibodies. The primary antibodies were as follows: NF- $\kappa \mathrm{B}$ p65 (1:1000, ab16502), p-NF- $\kappa \mathrm{B}$ p65 (1:500, ab86299), I $\kappa \mathrm{B} \alpha(1: 1000, \mathrm{ab} 32518), \mathrm{p}-\mathrm{I} \kappa \mathrm{B} \alpha(1: 500$, ab133462), NLRP3 (1:1000, ab214185), ASC ( $1: 1000$, ab155970), caspase-1 (1:1000, ab1872), and $\beta$-actin $(1: 2000, a b 8226)$, and were purchased from Abcam (Cambridge, UK). Afterward, the peroxidase-labeled secondary antibody (anti-rabbit IgG, 1:5000, Abcam) was used for incubation for $2 \mathrm{~h}$. Immunoreactive proteins were visualized with an enhanced chemiluminescence kit, and the signal densitometry was analyzed using a western blotting detection system (Quantity One, Bio-Rad, USA).

2.11. Statistical Analysis. Experimental data were expressed as the mean \pm standard deviation for normal distribution. Statistical analysis was performed using the SPSS 22.0 statistical software (Chicago, IL, USA). The statistical comparisons $(P$ values) were calculated using one-way analysis of variance or Student's $t$-test. If $P<0.05$, the difference was considered significant.

\section{Results}

3.1. AP Protects Mice against Sepsis-Induced ALI and Lung Edema. To determine whether AP could protect against sepsis-induced ALI, HE staining was performed. The results shown in Figure 1(a) indicated that pulmonary edema, hemorrhage, alveolar wall thickening, and inflammatory cell infiltration increased in the lungs of the CLP group mice compared to those of the lungs of the Sham group mice. In contrast, these histologic changes were attenuated by the administration of AP (Figure 1(a)). In addition, we assessed these changes by using the lung injury score and found that the administration of AP (50 and $100 \mathrm{mg} / \mathrm{kg}$ ) decreased the histological lung injury grade $(P<0.05, P<0.01)$, and the most effective AP concentration was $100 \mathrm{mg} / \mathrm{kg}$ (Figure 1(b)). To evaluate changes in lung edema, lung wet/dry weight ratios were determined. Figure 1(c) showed that the lung wet/dry weight ratio was higher in the CLP group than in the Sham group $(P<0.01)$. When compared with the CLP group, treatment with AP (50 and $100 \mathrm{mg} / \mathrm{kg}$ ) decreased the lung $\mathrm{W} / \mathrm{D}$ ratio $(P<0.05, P<0.01)$, and the most effective AP concentration was also $100 \mathrm{mg} / \mathrm{kg}$ (Figure 1(c)). Therefore, AP $(100 \mathrm{mg} / \mathrm{kg})$ was selected for further experiments. All the results indicated that AP could protect mice against sepsis-induced ALI and lung edema.

3.2. AP Inhibits Inflammatory Cell Infiltration in the BALF of Sepsis-Induced ALI Mice. To investigate the role of AP on lung inflammation, the number of inflammatory cells in BALF was counted. As Figures 2(a)-2(c) showed, the number 


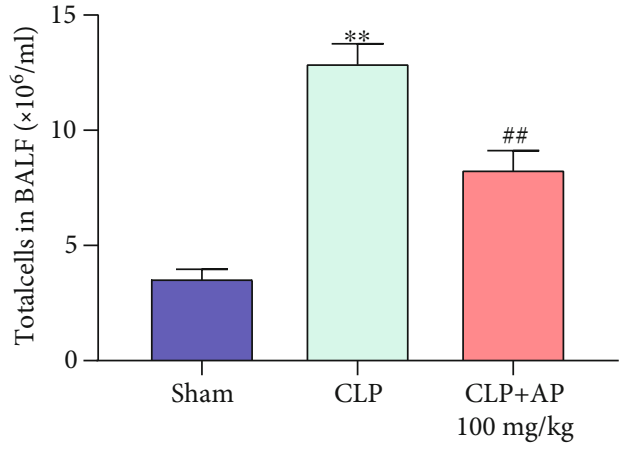

(a)

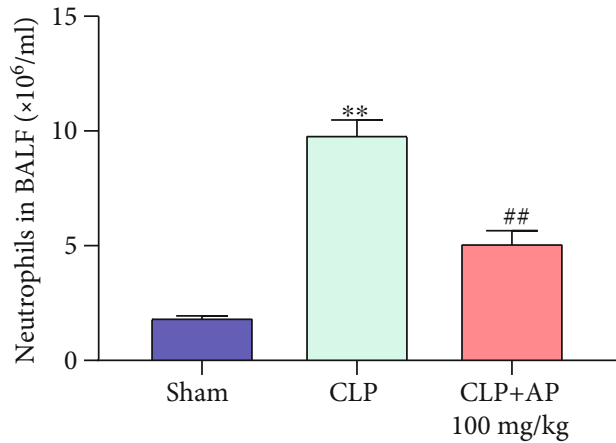

(b)

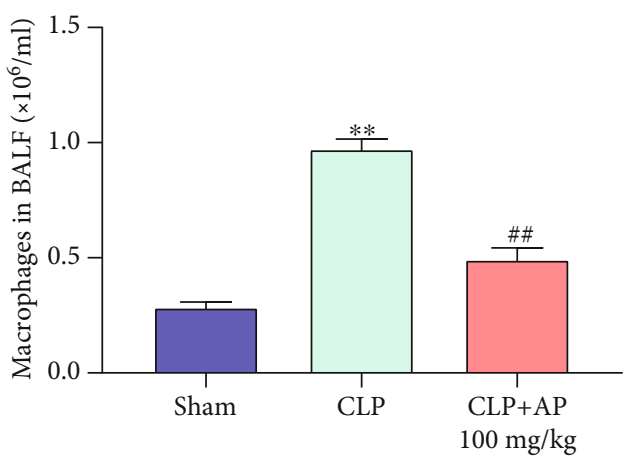

(c)

FIgURE 2: AP inhibited inflammatory cell infiltration in the BALF of sepsis-induced ALI mice. The number of total cells (a), neutrophils (b), and macrophages (c) was analyzed in BALF. ${ }^{* *} P<0.01$, vs. Sham group; ${ }^{\# \#} P<0.01$, vs. CLP group.

of total inflammatory cells, neutrophils, and macrophages increased in the CLP group compared to those of the Sham group $(P<0.01)$. When compared with the CLP group, AP treatment decreased the number of total inflammatory cells, neutrophils, and macrophages $(P<0.01)$, suggesting that AP could inhibit inflammatory cell infiltration in sepsisinduced ALI.

3.3. AP Inhibits the Production of Inflammatory Cytokines in Both the BALF and Serum of Sepsis-Induced ALI Mice. To evaluate the role of AP on inflammatory cytokines in sepsis-induced ALI mice, the levels of TNF- $\alpha$, IL- $1 \beta$, IL-6, and IL-18 in BALF and serum were detected by ELISA. The levels of TNF- $\alpha$, IL- $1 \beta$, IL- 6 , and IL-18 in the CLP group were higher than those in the Sham group $(P<0.01)$, while the levels of TNF- $\alpha$, IL- $1 \beta$, IL- 6 , and IL- 18 were reduced by AP treatment $(P<0.01)$ in BALF (Figures 3(a)-3(d)), as well as in serum (Figures $3(\mathrm{e})-3(\mathrm{~h})$ ). These results indicated that AP could inhibit the production of inflammatory cytokines in both the BALF and serum of sepsis-induced ALI mice.

3.4. AP Alleviates Oxidative Stress in Sepsis-Induced ALI Mice. In this study, we explored the antioxidative function of AP by measuring the activity of MPO, and the content of MDA, SOD, and GSH. The results demonstrated that the MPO activity and MDA content in the CLP group were higher than those in the Sham group $(P<0.01)$ (Figures 4(a) and 4(b)), while the levels of SOD and GSH were lower in the CLP group $(P<0.01)$ (Figures $4(\mathrm{c})$ and 4(d)). Conversely, the administration of AP decreased MPO activity and MDA content $(P<0.01)$ (Figures $4(\mathrm{a})$ and $4(\mathrm{~b}))$, and increased the levels of SOD and GSH $(P<0.01)$ (Figures 4(c) and 4(d)). These results indicated that AP could alleviate oxidative stress in sepsis-induced ALI.

3.5. AP Inhibits the Activation of the NF- $\kappa B$ Pathway in Sepsis-Induced ALI Mice. Western blot analysis showed that the expression of $\mathrm{p}-\mathrm{NF}-\kappa \mathrm{B}$ p 65 and $\mathrm{p}-\mathrm{I} \kappa \mathrm{B} \alpha$ was higher in the CLP group than in the Sham group $(P<0.01)$, but $\mathrm{I} \kappa \mathrm{B} \alpha$ expression was lower $(P<0.01)$ (Figure 5(a)). In contrast, the administration of AP decreased the expression of p-NF$\kappa \mathrm{B}$ p65 $(P<0.01)$ and $\mathrm{p}-\mathrm{I} \kappa \mathrm{B} \alpha(P<0.01)$, and increased $\mathrm{I} \kappa \mathrm{B} \alpha$ expression $(P<0.01)$ (Figure $5(\mathrm{a}))$. In addition, to further confirm the effect of AP on the NF- $\kappa$ B pathway, we measured p-NF- $\kappa$ B p65 expression in the lung tissue by immunohistochemistry. When compared with the Sham group, the protein expression of $\mathrm{p}-\mathrm{NF}-\kappa \mathrm{B}$ p65 increased in the CLP group $(P<0.01)$ (Figure 5(b)). Meanwhile, the phosphorylation of NF- $\kappa \mathrm{B}$ p65 was restrained by AP $(P<0.01)$ (Figure $5(\mathrm{~b})$ ). The results demonstrated that $\mathrm{AP}$ could inhibit the activation of the NF- $\kappa$ B pathway in sepsis-induced ALI mice.

3.6. AP Inhibits the Activation of the NLRP3 Inflammasome in Sepsis-Induced ALI Mice. As Figure 6(a) showed, the protein expression of NLRP3, ASC, and caspase-1 was upregulated in the CLP group relative to Sham group $(P<0.01)$. AP pretreatment inhibited the expression of NLRP3, ASC, and caspase-1 compared with the CLP group $(P<0.01)$ (Figure 6(a)). Moreover, we detected NLRP3 expression in the lung tissue by immunohistochemistry to better assess 


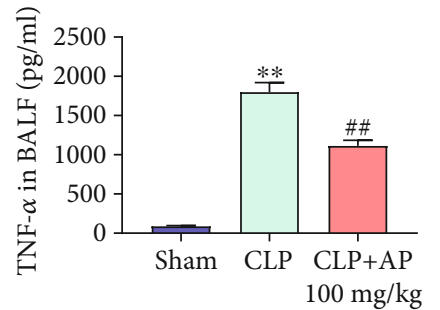

(a)

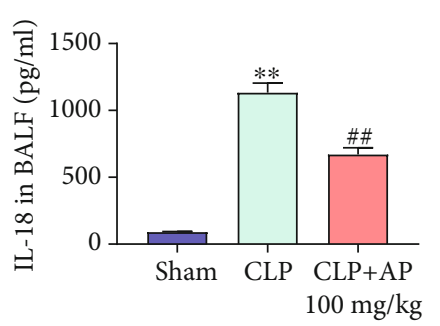

(d)

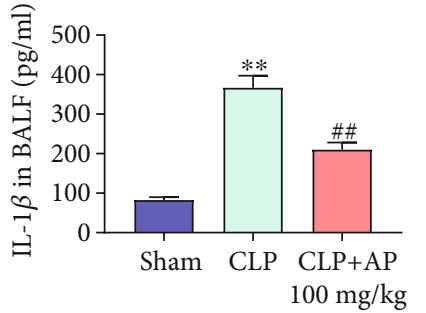

(b)

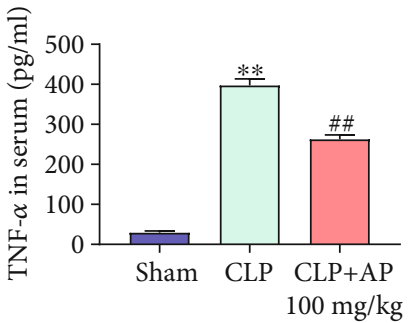

(e)

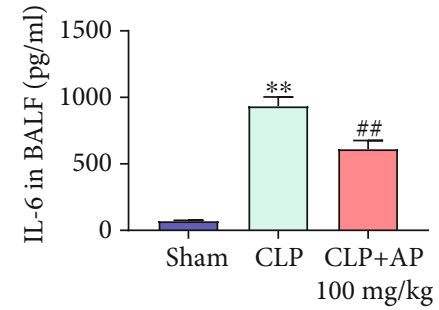

(c)

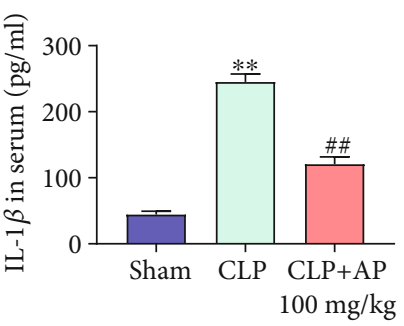

(f)

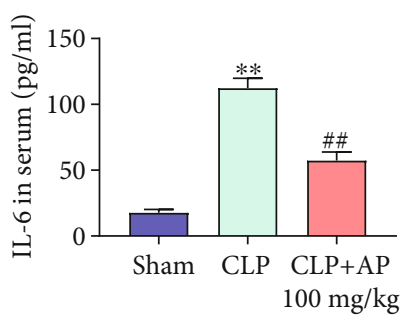

(g)

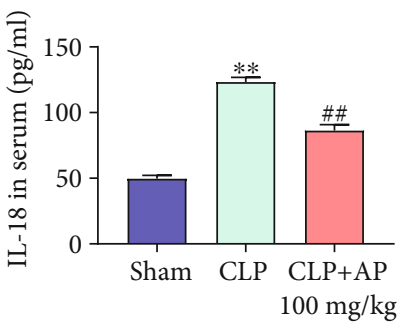

(h)

FIGURE 3: AP inhibited the production of inflammatory cytokines in both the BALF and serum of sepsis-induced ALI mice. The levels of TNF$\alpha$ (a), IL-1 $\beta$ (b), IL-6 (c), and IL-18 (d) in BALF were measured by ELISA. The levels of TNF- $\alpha$ (e), IL-1 $\beta$ (f), IL-6 (g), and IL-18 (h) in serum were measured by ELISA. ${ }^{* *} P<0.01$, vs. Sham group; ${ }^{\# \#} P<0.01$, vs. CLP group.

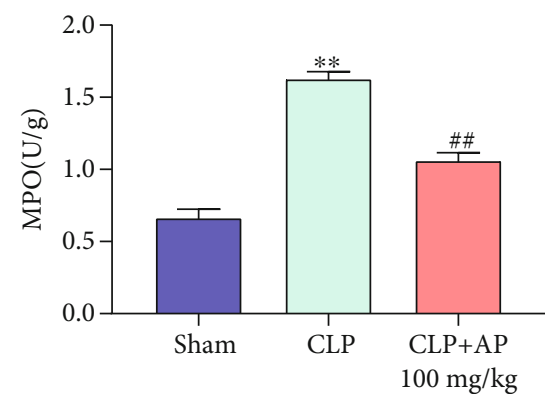

(a)

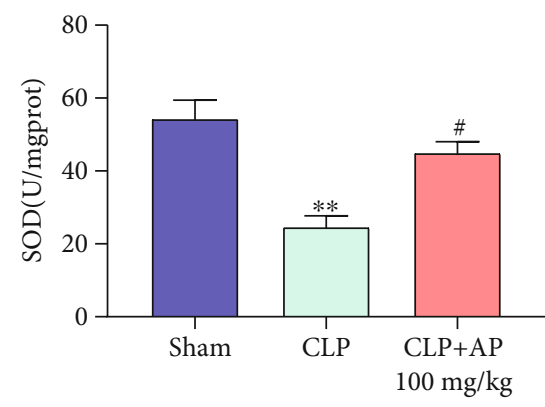

(c)

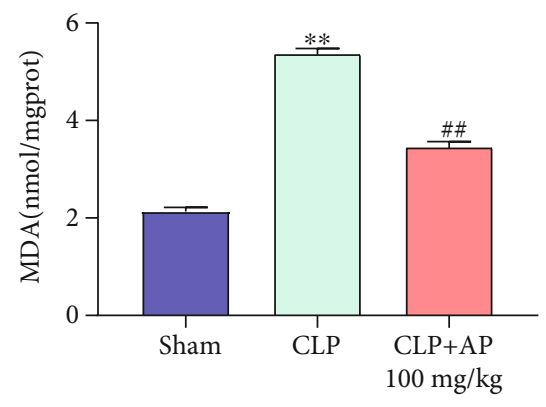

(b)

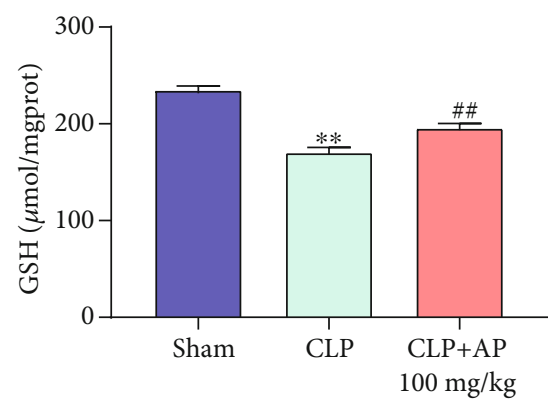

(d)

FIGURE 4: AP alleviated the oxidative stress in sepsis-induced ALI mice. The activity of MPO, MDA, SOD, and GSH was detected using the corresponding test kit. ${ }^{* *} P<0.01$, vs. Sham group; ${ }^{\# \#} P<0.01$, vs. CLP group. 


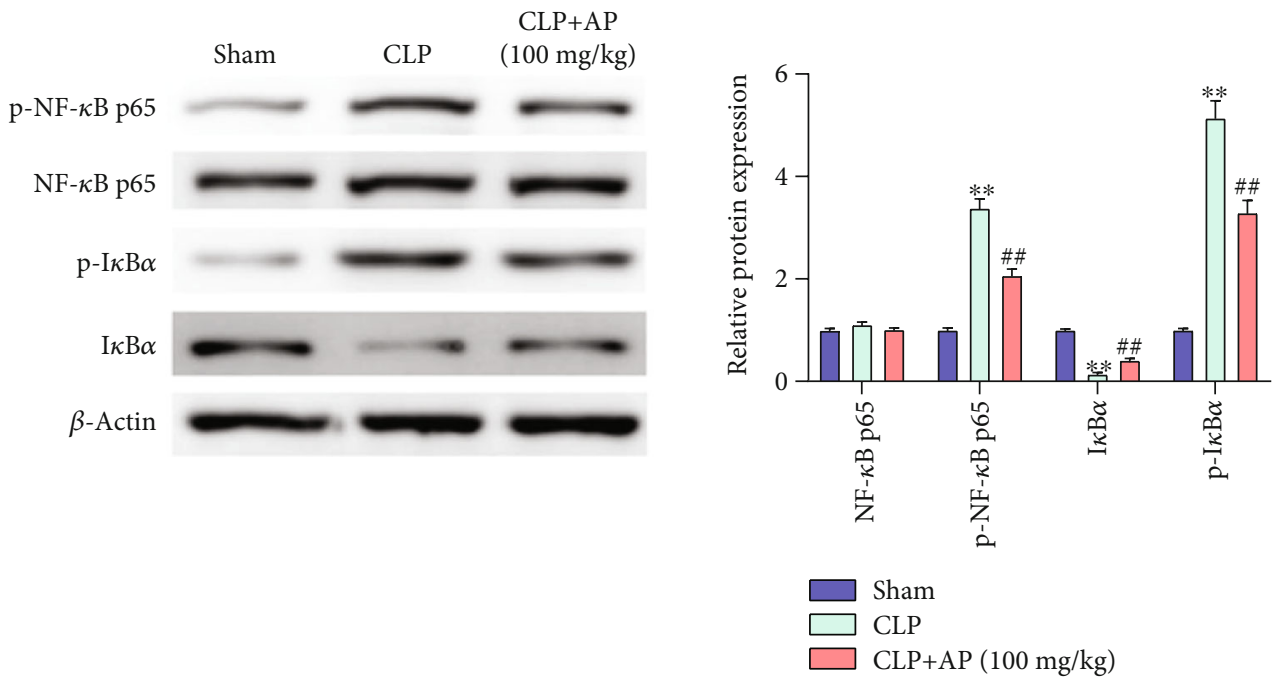

(a)
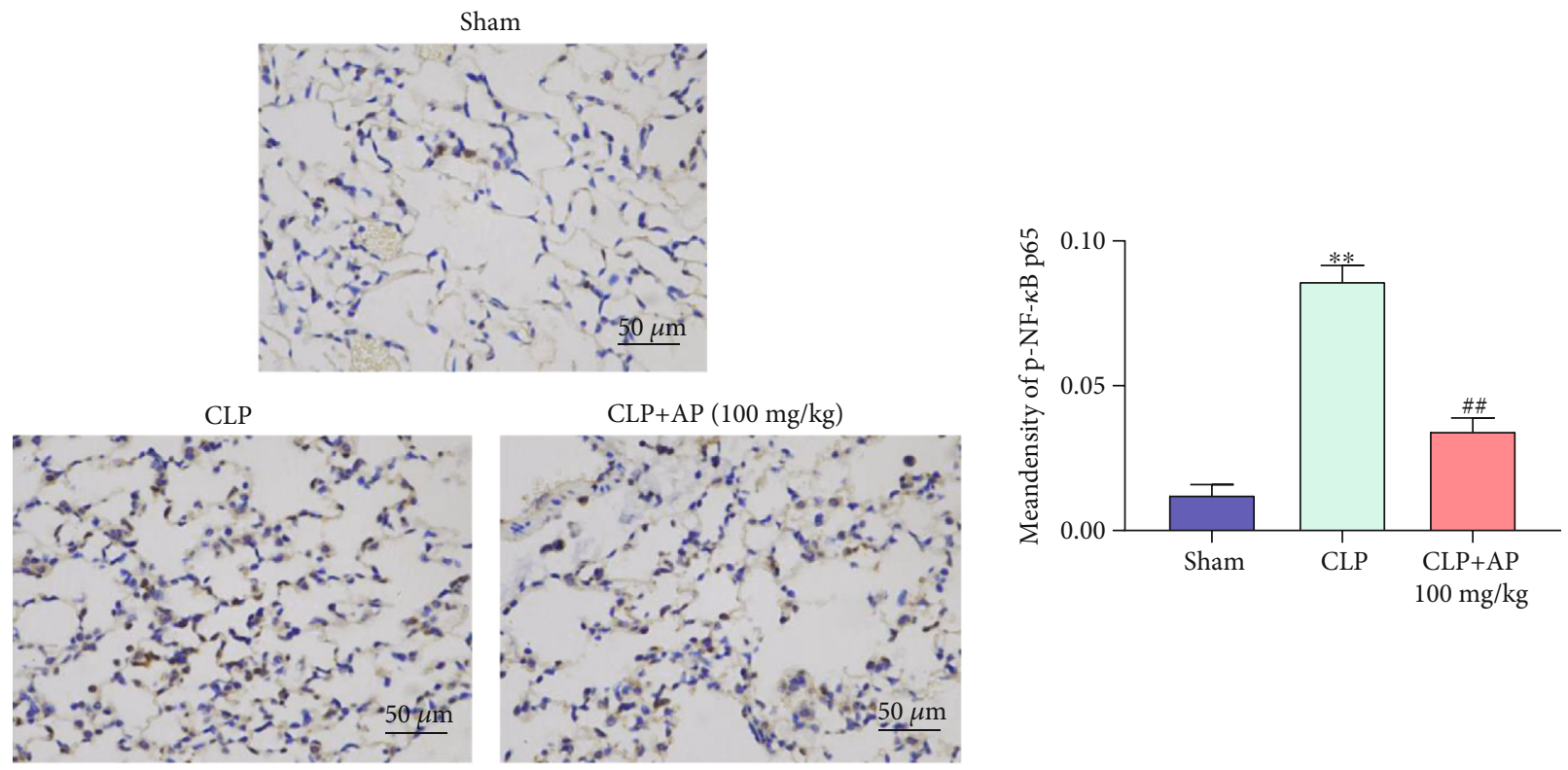

(b)

Figure 5: AP inhibited the activation of the NF- $\kappa$ B pathway in sepsis-induced ALI mice. (a) The expressions of NF- $\kappa \mathrm{B}$ p65, p-NF- $\kappa \mathrm{B}$ p65, $\mathrm{I} \kappa \mathrm{B} \alpha$, and $\mathrm{p}-\mathrm{I} \kappa \mathrm{B} \alpha$ were measured by western blot. (b) The expression of p-NF- $\kappa \mathrm{B}$ p 65 was detected by immunohistochemistry. ${ }^{* *} P<0.01$, vs. Sham group; ${ }^{\# \#} P<0.01$, vs. CLP group.

the effect of AP on the NLRP3 inflammasome. The results of Figure 6(b) demonstrated that the protein expression of NLRP3 was increased in the CLP group compared with the Sham group $(P<0.01)$. In contrast, the administration of AP decreased the protein expression of NLRP3 relative to the CLP group $(P<0.01)$ (Figure $6(\mathrm{~b})$ ). These results suggested that AP could inhibit the activation of the NLRP3 inflammasome in mice with sepsis-induced ALI.

\section{Discussion}

ALI is the injury of alveolar epithelial cells with high morbidity and mortality, which is related to some clinical disorders, including pneumonia and sepsis [17]. The five-year survival rate of patients with ALI is lower than 50\% [18]. Therefore, it is urgent to explore and discover new drug developments that possess high curative effects. In the present study, we demonstrated that AP could ameliorate sepsis-induced ALI in mice by inhibiting NLRP3 and NF- $\kappa$ B signaling pathways.

The representative symptoms of ALI are lung edema, inflammatory cell infiltration, and intrapulmonary hemorrhage [19]. Activated neutrophils and macrophages can directly induce endothelial injury and damage the basement membrane, leading to changes in vascular permeability and ultimately to pulmonary edema [20]. In the present study, AP treatment ameliorated the pathologic changes and decreased the W/D weight ratio in the lungs compared with those of the CLP group. In addition, we found that AP treatment reduced the number of inflammatory cells. The results indicated that AP could exert a protective effect against 


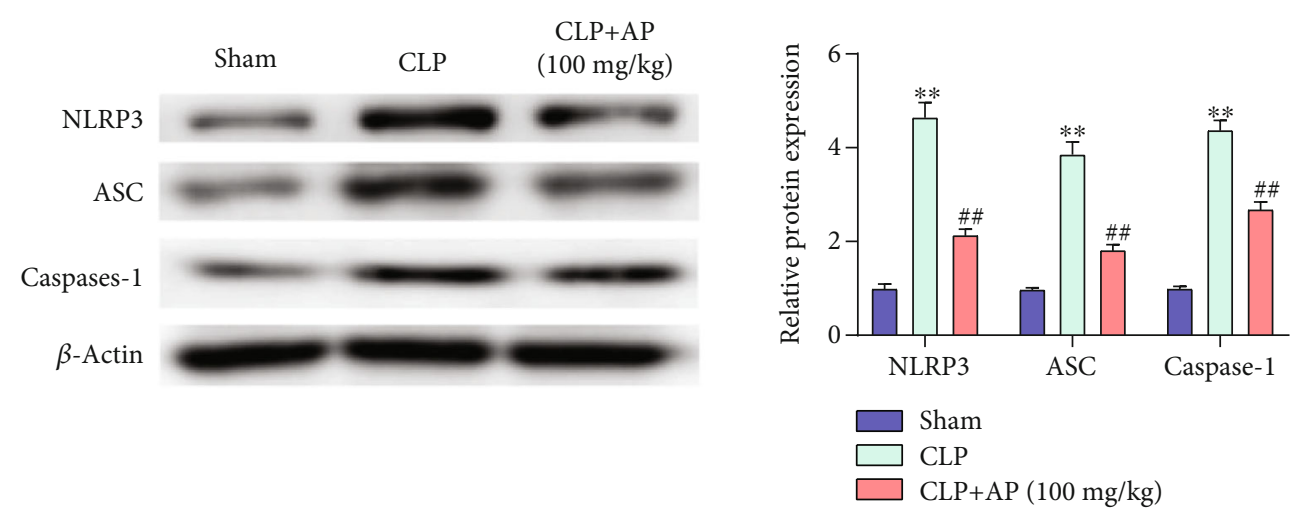

(a)
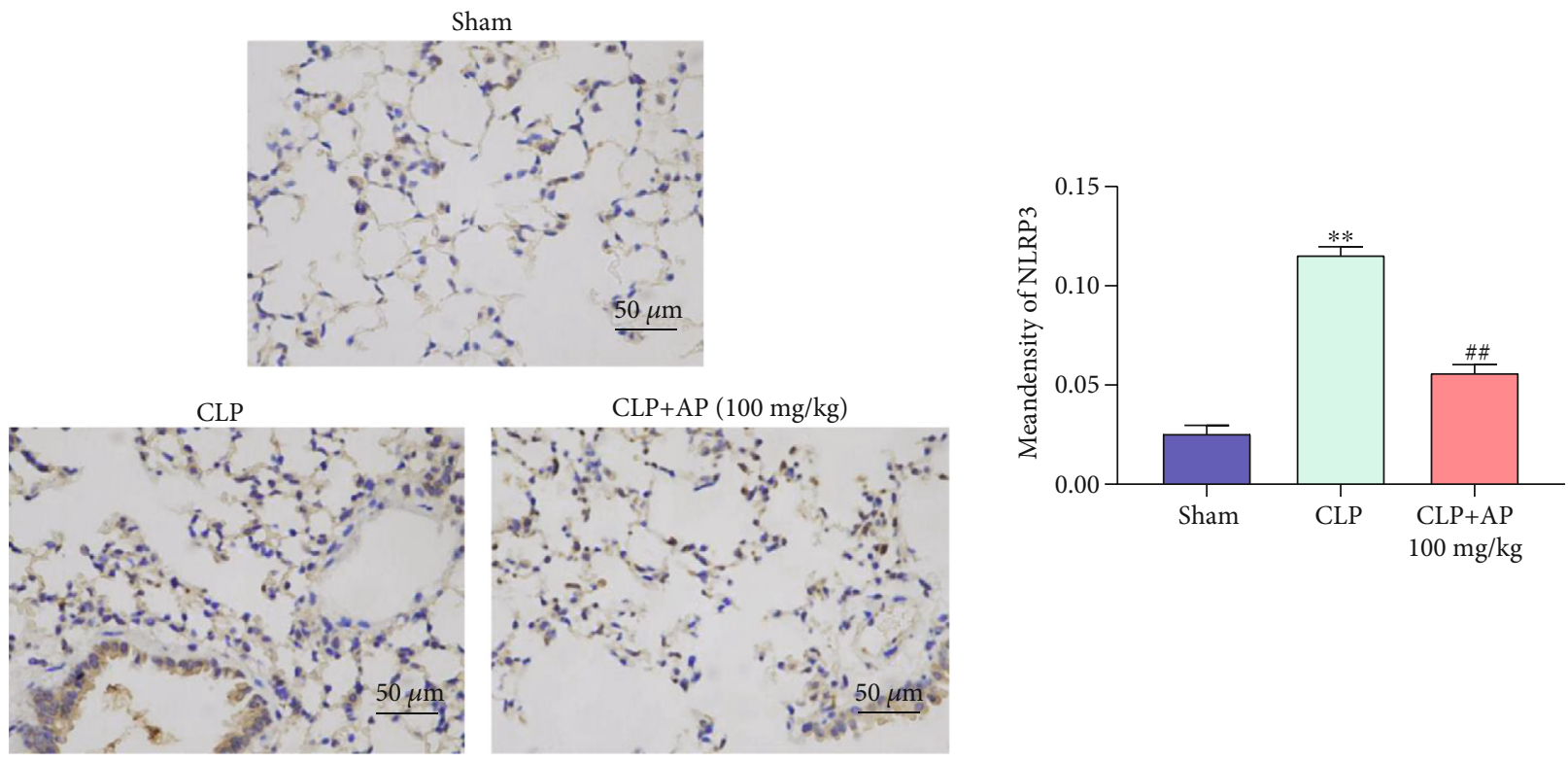

(b)

FIgURE 6: AP inhibited the activation of the NLRP3 inflammasome in sepsis-induced ALI mice. (a) The expressions of NLRP3, ASC, and caspase-1 were measured by western blot. (b) The expression of NLRP3 was detected by immunohistochemistry. ${ }^{* *} P<0.01$, vs. Sham group; ${ }^{\# \#} P<0.01$, vs. CLP group.

sepsis-induced ALI by ameliorating lung edema and inflammatory cell infiltration.

In recent years, an increasing number of studies have demonstrated that oxidative stress can induce the aggregation of inflammatory cells and cause a number of serious diseases, including ALI [21]. MPO is a marker of neutrophil accumulation, which plays an important role in the inflammatory response. Despite its positive effects on the organism, MPO-derived oxidants can cause oxidative stress and oxidative tissue damage [22]. MDA, a product of lipid peroxidation, is a marker of oxidative stress [23]. Previous studies have also reported that SOD and GSH are two essential antioxidant factors in the body [14]. In the present study, the administration of AP decreased MPO activity and MDA content, and increased the levels of SOD and GSH relative to the CLP group, suggesting that AP could alleviate the oxidative stress in sepsis-induced ALI. In addition to oxidative stress, inflammation is related to the pathogenesis of ALI. Inflammatory cytokines, such as TNF- $\alpha$, IL- $1 \beta$, IL-6, and IL-18, are reported to play important roles in the process of ALI $[24,25]$. The levels of TNF- $\alpha$, IL- $1 \beta$, IL- 6 , and IL-18 are upregulated in the serum and BALF of ALI mice [24, 26]. Similarly, we found that the levels of TNF- $\alpha$, IL- $1 \beta$, IL6 , and IL-18 were upregulated in the serum and BALF of sepsis-induced ALI mice, and the upregulation of these inflammatory cytokines was reversed by AP.

Among the various inflammatory responses, two pathways are particularly necessary for ALI: the NF- $\kappa \mathrm{B}$ and NLRP3 pathways. NF- $\kappa \mathrm{B}$ is a crucial nuclear transcription factor involved in the modulation of inflammatory cytokine production $[27,28]$. Under normal conditions, NF- $\kappa \mathrm{B}$ is sequestered into the cytoplasm by binding to $\mathrm{I} \kappa \mathrm{B} \alpha$, which is a representative member of $\mathrm{I} \kappa \mathrm{B}$ [29]. Upon stimulation, $\mathrm{I} \kappa \mathrm{B} \alpha$ is degraded and phosphorylated [30]. Subsequently, NF- $\kappa$ B is released and translocated to the nucleus where it binds to specific DNA to regulate inflammatory gene expression [30]. Our results showed that AP decreased the phosphorylation of NF- $\kappa \mathrm{B}$ p 65 and $\mathrm{I} \kappa \mathrm{B} \alpha$ and increased $\mathrm{I} \kappa \mathrm{B} \alpha$ expression, 
indicating that AP could inhibit the activation of the NF- $\kappa \mathrm{B}$ pathway in sepsis-induced ALI mice. An increasing number of studies have demonstrated that the NLRP3 inflammasome is involved in a variety of disease processes, including inflammation-related tissue injury [31], and that ROS generated from NF- $\kappa \mathrm{B}$-mediated inflammation is a danger signal that activates the NLRP3 inflammasome [32]. One activation, NLRP3 recruits ASC and activates caspase-1, which induces the secretion and maturation of IL- $1 \beta$ and IL-18 [12]. IL-1 $\beta$ and IL-18 further induce the release of other inflammatory factors, which mediate and expand the inflammatory cascade. Western blotting and immunohistochemistry showed that AP significantly decreased the expression of NLRP3, ASC, and caspase-1, suggesting that AP could inhibit the activation of the NLRP3 inflammasome in sepsis-induced ALI mice.

In conclusion, this study confirmed that AP could exhibit a protective effect against sepsis-induced ALI by inhibiting the NLRP 3 and NF- $\kappa$ B signaling pathways in mice. The findings of this study provide a new avenue for ALI and pave the way for future research.

\section{Data Availability}

The datasets used and analyzed during the current study are available from the corresponding author on reasonable request.

\section{Ethical Approval}

All studies involving animals were approved by the ethics committee of Zaozhuang Municipal Hospital (Jining medical college affiliated hospital) and conducted in accordance with the National Institutes of Health Guide for the Care and Use of Laboratory Animal.

\section{Conflicts of Interest}

The authors indicate no potential conflicts of interest.

\section{Authors' Contributions}

Dr. Yan Zhu, Dr. Taocheng Meng, and Dr. Aichen Sun contributed to study design, trial management, data interpretation, and writing the paper. Dr. Yan Zhu and Dr. Taocheng Meng contributed equally to the writing of this article. Dr. Jintao Li participated in study design, extracting and analysing the data, and writing the paper. Dr. Jinlai Li took part in extracting the data and modifying the paper.

\section{References}

[1] R. S. Hotchkiss, G. Monneret, and D. Payen, "Immunosuppression in sepsis: a novel understanding of the disorder and a new therapeutic approach," The Lancet Infectious Diseases, vol. 13, no. 3, pp. 260-268, 2013.

[2] G. S. Martin, D. M. Mannino, S. Eaton, and M. Moss, "The epidemiology of sepsis in the United States from 1979 through 2000," The New England Journal of Medicine, vol. 348, no. 16, pp. 1546-1554, 2003.
[3] F. Ren, J. Li, Y. Wang et al., "The effects of angelica sinensis polysaccharide on tumor growth and iron metabolism by regulating hepcidin in tumor-bearing mice," Cellular Physiology and Biochemistry, vol. 47, no. 3, pp. 1084-1094, 2018.

[4] P. Gu, S. Xu, S. Zhou et al., "Optimization of angelica sinensis polysaccharide-loaded Poly (lactic-co- glycolicacid) nanoparticles by RSM and its immunological activity in vitro," International Journal of Biological Macromolecules, vol. 107, pp. 222 229, 2018.

[5] Y. Xie, H. Zhang, Y. Zhang, C. Wang, D. Duan, and Z. Wang, "Chinese Angelica polysaccharide (CAP) alleviates LPSinduced inflammation and apoptosis by down-regulating COX-1 in PC12 cells," Cellular Physiology and Biochemistry, vol. 49, no. 4, pp. 1380-1388, 2018.

[6] W.-f. Zhang, Y. Yang, X. Li et al., "Angelica polysaccharides inhibit the growth and promote the apoptosis of U251 glioma cells_in vitro_and _in vivo_," Phytomedicine, vol. 33, pp. 2127, 2017.

[7] R. Li, F. Yin, Y. Guo, Q. Ruan, and Q. Zhu, “Angelica polysaccharide protects PC-12 cells from lipopolysaccharide-induced injury via down-regulating microRNA-223," Biomedicine \& Pharmacotherapy, vol. 108, pp. 1320-1327, 2018.

[8] J. Li, L. Li, S. Wang et al., "Resveratrol alleviates inflammatory responses and oxidative stress in rat kidney ischemiareperfusion injury and $\mathrm{H} 2 \mathrm{O} 2$-induced NRK-52E cells via the Nrf2/TLR4/NF- $\kappa$ B pathway," Cellular Physiology and Biochemistry, vol. 45, no. 4, pp. 1677-1689, 2018.

[9] K. Shimada, T. R. Crother, J. Karlin et al., “Oxidized mitochondrial DNA activates the NLRP3 inflammasome during apoptosis," Immunity, vol. 36, no. 3, pp. 401-414, 2012.

[10] R. de Martin, B. Vanhove, Q. Cheng et al., "Cytokine-inducible expression in endothelial cells of an I kappa B alpha-like gene is regulated by NF kappa B," The EMBO Journal, vol. 12, no. 7, pp. 2773-2779, 1993.

[11] S.-J. Kim and S.-M. Lee, "NLRP3 inflammasome activation in d-galactosamine and lipopolysaccharide- induced acute liver failure: Role of heme oxygenase-1," Free Radical Biology \& Medicine, vol. 65, pp. 997-1004, 2013.

[12] Z. Yang, J. Cao, C. Yu, Q. Yang, Y. Zhang, and L. Han, “Caspase-1 mediated interleukin-18 activation in neutrophils promotes the activity of rheumatoid arthritis in a NLRP3 inflammasome independent manner," Joint Bone Spine, vol. 83, no. 3, pp. 282-289, 2016.

[13] J. A. Jordan, R.-F. Guo, E. C. Yun et al., "Role of IL-18 in acute lung inflammation," Journal of Immunology, vol. 167, no. 12, pp. 7060-7068, 2001.

[14] X. Niu, L. Zang, W. Li et al., "Anti-inflammatory effect of Yam Glycoprotein on lipopolysaccharide-induced acute lung injury via the NLRP3 and NF- $\kappa$ B/TLR4 signaling pathway," International Immunopharmacology, vol. 81, article 106024, 2020.

[15] B. Zhang, B. Wang, S. Cao, Y. Wang, and D. Wu, "Silybin attenuates LPS-induced lung injury in mice by inhibiting NF- $\kappa \mathrm{B}$ signaling and NLRP3 activation," International Journal of Molecular Medicine, vol. 39, no. 5, pp. 1111-1118, 2017.

[16] H. Zhu, P. Luo, Y. Fu et al., "Dihydromyricetin prevents cardiotoxicity and enhances anticancer activity induced by adriamycin," Oncotarget, vol. 6, no. 5, pp. 3254-3267, 2015.

[17] W.-Y. Kim and S.-B. Hong, "Sepsis and acute respiratory distress syndrome: recent update," Tuberculosis and Respiratory Diseases, vol. 79, no. 2, pp. 53-57, 2016. 
[18] Q. Wang and L. Xiao, "Isochlorogenic acid a attenuates acute lung injury induced by LPS via Nf- $\kappa \mathrm{B} / \mathrm{NLRP} 3$ signaling pathway," American Journal of Translational Research, vol. 11, no. 11, pp. 7018-7026, 2019.

[19] M. A. Matthay, L. B. Ware, and G. A. Zimmerman, "The acute respiratory distress syndrome," vol. 122, no. 8, p. 2731, 2012.

[20] Y.-Y. Zhao, X.-P. Gao, Y. D. Zhao et al., "Endothelial cellrestricted disruption of FoxM1 impairs endothelial repair following LPS-induced vascular injury," Journal of Clinical Investigation, vol. 116, no. 9, pp. 2333-2343, 2006.

[21] M. J. Mondrinos, T. Zhang, S. Sun et al., "Pulmonary endothelial protein kinase C-delta (PKC $\delta$ ) regulates neutrophil migration in acute lung inflammation," The American Journal of Pathology, vol. 184, no. 1, pp. 200-213, 2014.

[22] E.-Y. Ko, S.-H. Cho, S.-H. Kwon et al., "The roles of NF- $\kappa$ B and ROS in regulation of pro-inflammatory mediators of inflammation induction in LPS-stimulated zebrafish embryos," Fish \& Shellfish Immunology, vol. 68, pp. 525-529, 2017.

[23] D. Manca, A. C. Ricard, B. Trottier, and G. Chevalier, "Studies on lipid peroxidation in rat tissues following administration of low and moderate doses of cadmium chloride," Toxicology, vol. 67, no. 3, pp. 303-323, 1991.

[24] S. Yang, Z. Yu, L. Wang et al., "The natural product bergenin ameliorates lipopolysaccharide-induced acute lung injury by inhibiting NF-kappaB activition," Journal of Ethnopharmacology, vol. 200, pp. 147-155, 2017.

[25] T. Hoshino, M. Okamoto, Y. Sakazaki, S. Kato, H. A. Young, and H. Aizawa, "Role of proinflammatory cytokines IL-18 and IL-1 $\beta$ in bleomycin-induced lung injury in humans and mice," American Journal of Respiratory Cell and Molecular Biology, vol. 41, no. 6, pp. 661-670, 2009.

[26] L. Tian, W. Li, and T. Wang, "Therapeutic effects of silibinin on LPS-induced acute lung injury by inhibiting NLRP3 and NF- $\kappa \mathrm{B}$ signaling pathways," Microbial Pathogenesis, vol. 108, pp. 104-108, 2017.

[27] W.-C. Huang, C.-L. Lai, Y.-T. Liang, H.-C. Hung, H.-C. Liu, and C.-J. Liou, "Phloretin attenuates LPS-induced acute lung injury in mice via modulation of the NF- $\kappa$ B and MAPK pathways," International Immunopharmacology, vol. 40, pp. 98105, 2016.

[28] G. He, X. Zhang, Y. Chen, J. Chen, L. Li, and Y. Xie, "Isoalantolactone inhibits LPS-induced inflammation via $\mathrm{NF}-\kappa \mathrm{B}$ inactivation in peritoneal macrophages and improves survival in sepsis," Biomedicine \& Pharmacotherapy, vol. 90, pp. 598607, 2017.

[29] M. S. Hayden and S. Ghosh, "Signaling to NF- $\kappa$ B," Genes \& Development, vol. 18, no. 18, pp. 2195-2224, 2004.

[30] C. M. Alvira, "Nuclear factor-kappa-B signaling in lung development and disease: one pathway, numerous functions," Birth Defects Research Part A: Clinical and Molecular Teratology, vol. 100, no. 3, pp. 202-216, 2014.

[31] Y. Zhang, X. Li, J. J. Grailer et al., "Melatonin alleviates acute lung injury through inhibiting the NLRP3 inflammasome," Journal of Pineal Research, vol. 60, no. 4, pp. 405-414, 2016.

[32] F. G. Bauernfeind, G. Horvath, A. Stutz et al., "Cutting edge: NF- $\kappa \mathrm{B}$ activating pattern recognition and cytokine receptors license NLRP3 inflammasome activation by regulating NLRP3 expression," The Journal of Immunology, vol. 183, no. 2, pp. 787-791, 2009. 